Article

\title{
Analysis of the Thermal Decomposition Products of Pathological and Healthy Tissues in Paranasal Sinuses: A High-Resolution Terahertz Gas Spectroscopy Study
}

\author{
Vladimir Vaks ${ }^{1,2, *}$, Andrey Aizenshtadt ${ }^{1,3}$, Vladimir Anfertev ${ }^{1}$, Maria Chernyaeva ${ }^{1,2}\left(\mathbb{D}\right.$, Elena Domracheva ${ }^{1}(\mathbb{D}$, \\ Kseniya Gavrilova ${ }^{1,3}$, Roman Larin ${ }^{1,4}$, Sergey Pripolzin ${ }^{1}$ and Mariya Shakhova ${ }^{1,5}$ \\ 1 Institute for Physics of Microstructures of RAS, 603950 Nizhny Novgorod, Russia; sleepred@yandex.ru (A.A.); \\ anfertev@ipmras.ru (V.A.); masha@ipmras.ru (M.C.); elena@ipmras.ru (E.D.); \\ super.gavrilova-kseniy@yandex.ru (K.G.); info.lor@mail.ru (R.L.); psi@ipmras.ru (S.P.); \\ maha-shakh@yandex.ru (M.S.) \\ 2 Radiophysics Faculty, Lobachevsky State University, 603950 Nizhny Novgorod, Russia \\ 3 Children Clinical Hospital No.1, 603950 Nizhny Novgorod, Russia \\ 4 State Budgetary Healthcare Institution “N.A. Semashko Regional Clinical Hospital”, \\ 603950 Nizhny Novgorod, Russia \\ 5 General Medicine Department, Privolzhsky Research Medical University, 603950 Nizhny Novgorod, Russia \\ * Correspondence: vax@ipmras.ru; Tel.: +7-831-4179457
}

Citation: Vaks, V.; Aizenshtadt, A.; Anfertev, V.; Chernyaeva, M.; Domracheva, E.; Gavrilova, K.; Larin, R.; Pripolzin, S.; Shakhova, M. Analysis of the Thermal

Decomposition Products of Pathological and Healthy Tissues in Paranasal Sinuses: A High-Resolution Terahertz Gas Spectroscopy Study. Appl. Sci. 2021, 11, 7562. https:// doi.org/10.3390/app11167562

Academic Editor: Renato Torre

Received: 12 July 2021

Accepted: 16 August 2021

Published: 18 August 2021

Publisher's Note: MDPI stays neutral with regard to jurisdictional claims in published maps and institutional affiliations.

Copyright: (c) 2021 by the authors. Licensee MDPI, Basel, Switzerland. This article is an open access article distributed under the terms and conditions of the Creative Commons Attribution (CC BY) license (https:// creativecommons.org/licenses/by/ $4.0 /)$.

\begin{abstract}
We present the novel results of studying tissue metabolites of the ear-nose-throat organs (pathological and relatively healthy mucosal tissues) during heating and thermal exposure using gas spectrometers of the terahertz $(\mathrm{THz})$ frequency range based on non-stationary effects. Tissue metabolites of the ear-nose-throat organs obtained during surgery according to strict medical indications have been studied for the first time in the world. All the tissues used in the samples were histological verified for confirming the morphological diagnosis. For obtaining a unified picture, chemical compounds appearing during thermal decomposition of pathological and relatively healthy tissues were identified using the spectroscopic approach, with mandatory histological verification of the samples. The obtained results demonstrate that a powerful research tool has been proposed for evaluation of metabolites in otorhinolaryngology with detection of diseases markers.
\end{abstract}

Keywords: THz high resolution spectroscopy; ENT-organs; metabolite; thermal decomposition; relatively healthy nasal mucosa; polyp

\section{Introduction}

Chronic inflammatory and systemic diseases affecting the ear-nose-throat (ENT) organs are different in their clinical course and etiology. However, there are a number of common properties and features in the course of diseases due to a common morphological substratum for the development of inflammation. The anatomical peculiarity of the structure of the ENT organs, due to the isolation of the cavities, has always set intricate problems for scientists investigating these organs. At the same time, the relative isolation was attractive for studying the processes in the ENT organs, including inflammatory ones, since the relative isolation excluded the influence of other organs and systems of the human body and provided a relative purity of the experiment in the considered object.

One of the research objects of interest for otorhinolaryngology is the development of a precision method that enables assessment of metabolites in the ENT organs with detection of diseases markers.

A consensus document EPOS 2020 on modern approaches to the diagnosis and treatment of rhinosinusitis, including chronic ones (CRS), was published in 2020 [1]. In this document, definite aspects of the main mechanisms of the development of this pathology 
are identified and ways to improve diagnostic criteria for understanding the etiology and pathogenesis of the disease are outlined [2].

The CRS pathogenesis is based on the processes of the interaction of environmental factors and mucosa of the nasal cavity and sinuses, in which, in the presence of a defect in the mucociliary transport functioning and defects in the functioning of local adaptive immunity, an inflammatory cascade is triggered. This inflammatory cascade is based on the so-called endotypes, depending on the immune cells and pro-inflammatory mediatorscytokines involved in the pathological cascade: T2-mechanisms caused by the activation of Th2-lymphocytes, congenital lymphoid cells of the second type, as well as some other cells of the immune system and the production of T2-cytokines leading to the development of a systemic inflammatory process, and non-T2 mechanisms. Different inflammatory endotypes trigger pathological remodeling processes in the mucosa, which, in turn, leads to the development of inflammatory phenotypes: nasal polyposis, oteitis and so on [3-6].

One of the innovative approaches to the study of the etiology and pathogenesis of diseases and systemic pathologies is based on metabolomics. The objects of metabolomics studies are intermediate and final metabolites formed as a result of metabolism in the biological system $[7,8]$. Conventionally, the objects for analysis include exhaled breath, biological liquids, pathological secretions, and, to a lesser extent, the contents of inflamed areas, tissue biopsies due to the difficulty of analysis and the lack of research methods. In 2004, the Human Metabolome Project (HMP) (Edmonton, AB, Canada) was initiated aimed at creating a database of final or intermediate metabolic products in the human organism. A public electronic Human Metabolome Database (HMDB) was set up [9]. At present, it contains data on more than 114,020 metabolites of the human organism detected in blood, urine, sweat, etc. The metabolites were detected mainly by mass spectrometry and gas chromatography, as well as by spectroscopic methods, for example, in the IR range.

Currently there is no database on tissue metabolites or on pathological secretion obtained from inflamed areas and tumors. Some information, and only for some substances as markers of the pathological process, can be found from the Human Metabolom Database. Studies of tissue metabolites aimed at developing a metabolic profile and detecting markers, i.e., substances representing a particular object under study, will make it possible to reveal pathogenetic mechanisms of disease development and application points during therapy.

The molecular absorption spectroscopy, in particular spectroscopy based on nonstationary effects (the appearance and decomposition of macroscopic polarization) is an effective approach to the study of multicomponent gas mixtures. Non-stationary spectrometers have the best approximation to the theoretical sensitivity limit (the sensitivity for some gases is about $0.2 \mathrm{ppb}$ in the scanning mode with a significant decrease in gas pressure (down to hundredths and thousandths of a torr)) with a resolution limited only by the Doppler effect [10]. The use of the terahertz (THz) frequency range, where the most intense absorption lines of the rotational spectrum of molecules, including organic ones, lie, makes this approach convenient for studying multicomponent gas mixtures of different origins, including biological ones [11,12]. By detecting the absorption lines in the spectra obtained during radiation passage through a sample that is a multicomponent gas mixture, it is possible to identify differences in the composition of samples corresponding to different pathologies, and, consequently, potential markers of pathologies and diseases.

The objective of the study was to use spectrometers based on high-resolution nonstationary $\mathrm{THz}$ spectroscopy methods to explore the composition of metabolites in the products of thermal decomposition of healthy tissue and a characteristic set of metabolites aimed to detect markers of inflammation in a pathology such as chronic polypous rhinosinusitis.

\section{Materials and Methods}

The method of $\mathrm{THz}$ spectroscopy based on the effect of freely decaying polarization works as follows. If a polarization is created in a gas sample under resonant conditions, then after the oscillator is pulled out of resonance, the polarization wave will emit the same 
field mode that excited it. This is done by periodically switching the phase or frequency of the probe radiation resonantly interacting with the medium, which leads to non-stationary radiation and absorption, i.e., to the periodic appearance and decay of induced macroscopic polarization. For this we have developed a spectrometer with phase switching of radiation and a fast frequency sweep spectrometer [10]. The phase switching mode [13] allows measurements to be performed at a particular frequency and is needed to monitor the dynamics of the selected substance concentration.

The principle of operation of the spectrometer with phase switching of the radiation acting on the gas (the block diagram is shown in Figure 1) is as follows: the frequency of the radiation source (RS) is modulated using a phase modulator (PM) and is set by the frequency of the frequency synthesizer (FS) preset by a computer (PC). Passing through the cell with the gas under study and interacting with the gas absorption line, the radiation enters the radiation detector (RD), where it is detected, and then amplified by an amplifier (AMP). After conversion from analog to digital with using an analog-to-digital converter (ADC), the signal containing information about the gas absorption line is accumulated in the storage device and then detected and processed in the computer. There are two possible ways to detect the absorption line:

- $\quad$ To detect the absorption line amplitude and the change in this amplitude over time by selecting one frequency (the absorption line center);

- To tune the frequency of the radiation source in a needed range and detect the absorption lines that lie within this range.

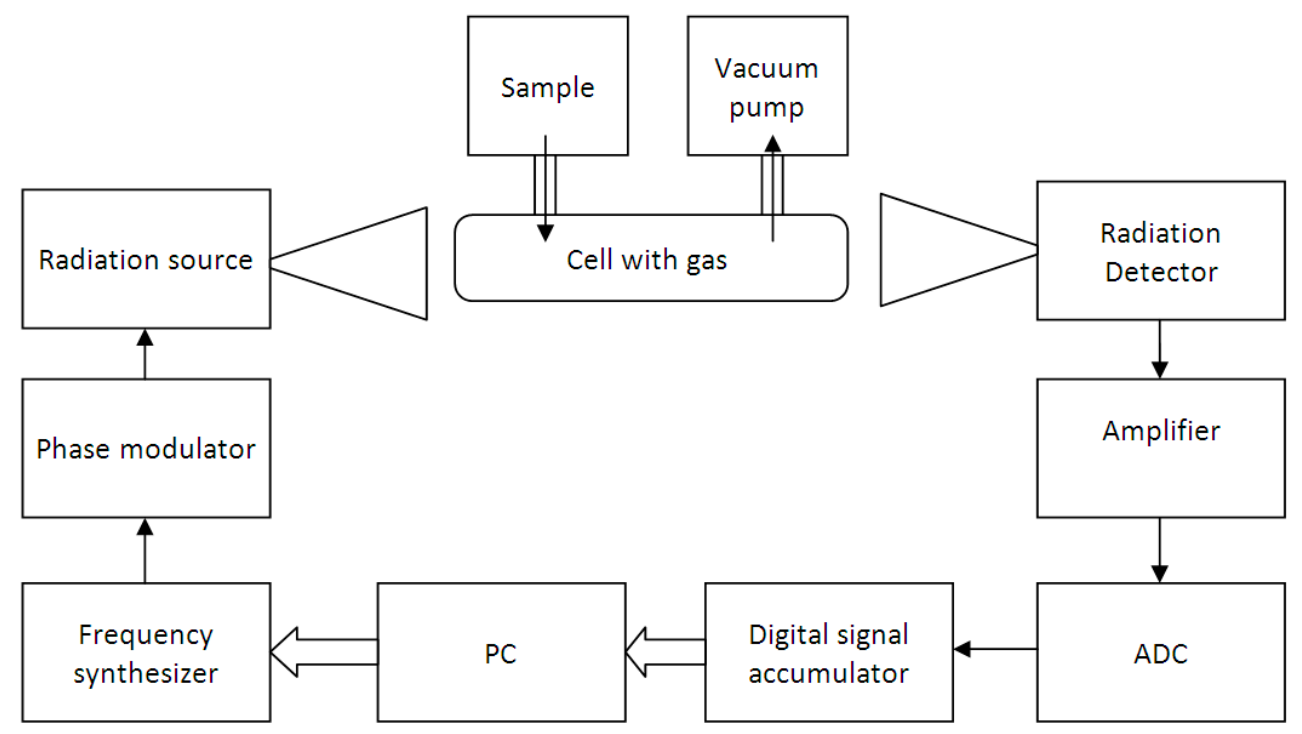

Figure 1. Block diagram of a spectrometer with phase switching of the radiation source signal.

In the first case the sensitivity of the spectrometer with phase switching of the radiation acting on the gas amounts to $5 \times 10^{-10} \mathrm{~cm}^{-1}$ and in the second case is $3 \times 10^{-9} \mathrm{~cm}^{-1}$.

Another approach is fast frequency passage (sweep) through the absorption line [14]. Fast sweep makes it possible to detect all absorption lines in the entire frequency range of the spectrometer and determine the presence of several substances at once. A block diagram of a fast frequency sweep spectrometer is shown in Figure 2.

The signal of the fast frequency sweep source is transferred to the frequency range of the radiation source (RS) using a broadband phase lock-loop (PLL) system. The frequency ranges are PC controlled. The radiation passes through a cell with a sample of the gas under study, interacts with it and enters the radiation detector (RD). The signal from the $\mathrm{RD}$ is amplified by the amplifier (AMP) and converted to digital form by an analog-todigital converter (ADC). This signal contains information about all the absorption lines of substances in the studied gas mixture, which lie in the sweep frequency range. Further, 
the digital signal is accumulated in the digital storage accumulator (DSA) to reduce the receiver noise and thereby increase the spectrometer sensitivity. From the DSA the signal is forwarded to the PC, where it is processed and further accumulated.

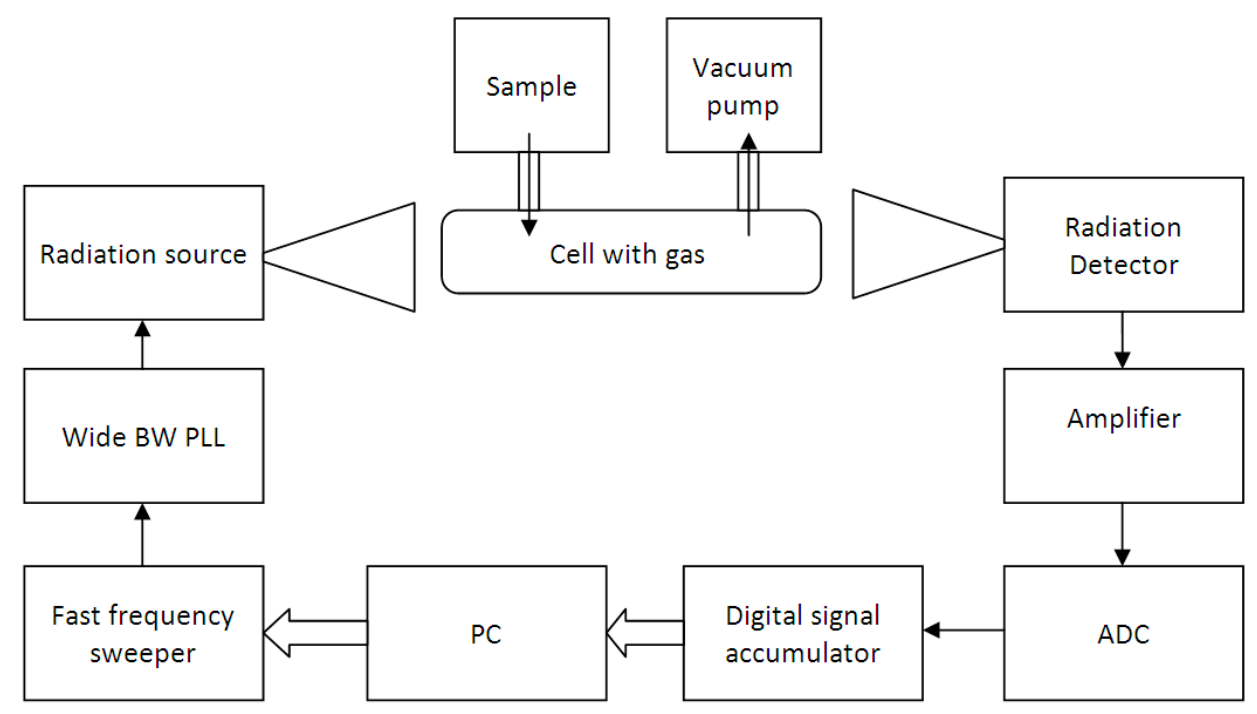

Figure 2. Frequency sweep spectrometer.

It takes the spectrometer $30 \mathrm{~s}$ to cover the frequency range of $60 \mathrm{GHz}$. The sensitivity of the recorded absorption coefficient for a cell length of $1 \mathrm{~m}$ is from $10^{-7} \mathrm{~cm}^{-1}$ to $5 \times 10^{-8} \mathrm{~cm}^{-1}$.

Using the method of $\mathrm{THz}$ non-stationary spectroscopy, we studied the composition of thermal decomposition products for samples of tissues of the nasal cavity and paranasal sinuses in norm and pathology, taken from patients during surgery performed according to strict medical indication, based on the functional endoscopic sinus surgery (FESS) principles. The age of patients was from 8 up to 45 years. Fragments of relatively healthy mucosa (Samples 1-3) and polyps of the nasal cavity and paranasal sinuses (Samples 4-6) were studied. To obtain a unified picture we identified metabolites in the study of pathological and relatively healthy tissue with mandatory histological verification of the samples.

Fragments of relatively healthy mucosa were obtained as a result of the surgical access, following to the FESS principles. Histological analysis verified the respiratory epithelium, that is a form of multilayered columnar epithelium. A large number of ciliated and goblet cells, as well as single isolated inflammation cones were observed. This pattern corresponded to the areas of relatively healthy mucosa.

The histomorphological assessment of polyposis changes in the mucosa revealed pronounced eosinophilic inflammation, slight neutrophilic infiltration, areas of fibrosis, and pronounced subepithelial edema of the stroma.

All samples had a relatively equal volume, were placed in $5 \mathrm{~mL}$ of distilled water for preservation and were delivered to the laboratory for spectroscopic analysis.

The sample was placed in a test tube connected to a pre-evacuated cell with a pressure of $5 \times 10^{-4}$ mbar. The test tube with the sample was heated (to temperatures of $200-250{ }^{\circ} \mathrm{C}$ ) and a mixture of thermal decomposition products of the sample was injected into the cell. The working pressure in the cell was $5 \times 10^{-2}$ mbar. The measurements were performed with the use of a spectrometer of the 118-178 GHz range operating in the phase switching mode, as well as with a fast frequency sweep spectrometer in the 118-178 GHz range. The substances were identified using electronic databases [15,16]. A spectrometer with phase switching of the radiation acting on the gas makes it possible to detect weaker absorption lines, and, consequently, to track lower concentrations of substances in the mixture, but the process of recording a range section is slower. A fast sweep frequency spectrometer enables 
one to quickly (within $30 \mathrm{~s}$ ) register the entire operating range of the spectrometer, i.e., it makes it possible to detect changes in the composition of substances in the mixture and to monitor the qualitative changes in their concentration. The study of samples using both spectrometers give a more complete picture of the thermal decomposition of a biological sample and reveals the most complete composition of a multicomponent gas mixture of thermal decomposition products of biological tissues, including the dynamics of the appearance of components.

The frequency range in which the studies were performed was always chosen the same for a spectrometer with phase switching of the radiation acting on the gas. Studies with the fast frequency sweep spectrometer were performed in the entire operating frequency range. Therefore, at the same operating pressure, it is possible to see a change in the concentration of substances included in the gas mixture of thermal decomposition products of tissues, by increasing the amplitude of the signal from the absorption line, and, consequently, increasing the absorption coefficient, and the appearance of weaker lines of the same substances that were not visible at lower concentrations. Thus, it is possible to make a qualitative comparison of the concentrations of substances by comparing the number of observed lines registered by the same spectrometer.

\section{Results}

Samples of relatively healthy mucosa and tissue samples of nasal cavity polyps and paranasal sinuses have been studied.

The comparison of substances revealed in the spectra of thermal decomposition products of relatively healthy tissue samples and polypous tissue samples some characteristic substances that have intense absorption lines in the operating ranges of the used spectrometers and appear in the gas mixture during thermal decomposition of the samples in a sufficiently large amount. The spectral data for the absorption lines of all the studied substances are available on-line in the databases [15,16].

One of these substances is acetaldehyde. It has a whole series of lines (over 900) with an integral intensity greater than $10^{-6} \mathrm{~nm}^{2} \mathrm{MHz}$ in the range of $118-178 \mathrm{GHz}$ [15]. Since the thermal decomposition products are a mixture of substances in the gas phase, the concentrations of the substances that form it are quite low. Therefore, the most intense absorption lines of a particular substance, including acetaldehyde, are observed in the spectrum. An example of recording a spectrum part containing lines of acetaldehyde appearing during thermal decomposition of both relatively healthy mucosa (No. 2) and polyp tissue (No. 6) is shown in Figure 3.

We have performed a comparative analysis of the data and their schematic interpretation as line diagrams. An example of the chemical composition of the products of thermal decomposition of a relatively healthy nasal mucosa for three samples (Samples 1-3) is shown in Figure 4.

We can see in the above diagrams that some metabolites are repeated and can be regarded as markers creating the so-called metabolic profile of a healthy tissue of the nasal mucosa and paranasal sinuses. These are, first of all, acetaldehyde, as well as methyl mercaptan, azole, butyronitrile, benzonitrile, ethylene sulfide, and carbonyl sulfide.

The spectroscopic study of the pathological process, in which substances differ significantly in concentration from those present in relatively healthy tissue, or absent in it are identified, makes it possible to conclude that there are decay products that characterize a particular pathology. An example of a substance that does not appear in all samples of relatively healthy tissue (only in one sample out of three, shown in Figure 4), is acetonitrile. However, acetonitrile is present in all the considered samples of polyp tissue. An example of recording a spectrum section containing acetonitrile $\left(\mathrm{CH}_{3} \mathrm{CN}\right)$ lines for a polyp tissue sample (No. 6) is shown in Figure 5. 


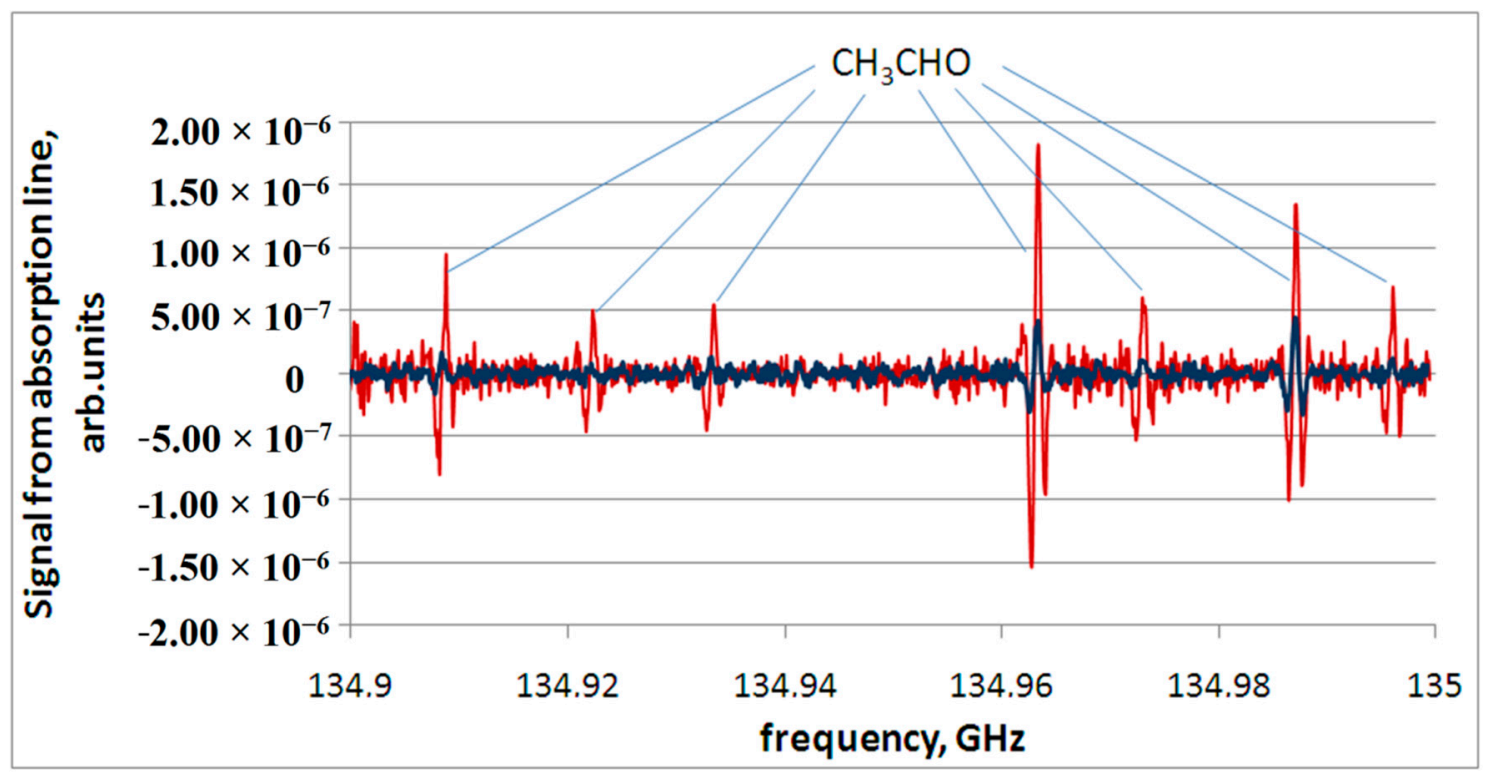

Figure 3. Example of recording a spectrum section containing acetaldehyde $\left(\mathrm{CH}_{3} \mathrm{CHO}\right)$ lines for Sample No. 2 (blue line) and Sample No.6 (red line) with experimentally measured central frequencies $\mathrm{f}_{1 \exp }=134.9087 \mathrm{GHz}$ (cf. with the frequency from on-line database $\mathrm{f}_{11 \text { cat }}=134.9084537 \mathrm{GHz}$ for the transition with quantum numbers $7440 \leftarrow 64$ 30 [15], $\mathrm{f}_{12 \text { cat }}=134.9086558 \mathrm{GHz}$ for the transition with quantum numbers $7430 \leftarrow 6420$ [15]), $\mathrm{f}_{2 \exp }=134.9222 \mathrm{GHz}$ $\left(f_{2 \text { cat }}=134.9222164 \mathrm{GHz}\right.$ for the transition with quantum numbers $\left.7432 \leftarrow 6422[15]\right), f_{3 \exp }=134.9334 \mathrm{GHz}$ $\left(f_{3 \text { cat }}=134.9333724 \mathrm{GHz}\right.$ for the transition with quantum numbers $74441 \leftarrow 6431$ [15]), $\mathrm{f}_{4 \exp }=134.9633 \mathrm{GHz}$ $\left(f_{4 \text { cat }}=134.9632399 \mathrm{GHz}\right.$ for the transition with quantum numbers $\left.73350 \leftarrow 63400[15]\right), f_{5 \exp }=134.9729 \mathrm{GHz}$ $\left(\mathrm{f}_{5 \text { cat }}=134.9730698 \mathrm{GHz}\right.$ for the transition with quantum numbers $7342 \leftarrow 6332$ [15]), $\mathrm{f}_{6 \exp }=134.98705 \mathrm{GHz}$ $\left(f_{6 c a t}=134.9872121 \mathrm{GHz}\right.$ for the transition with quantum numbers $7340 \leftarrow 6330$ [15]) and $\mathrm{f}_{7 \exp }=134.996 \mathrm{GHz}$ $\left(f_{7 \text { cat }}=134.9960633 \mathrm{GHz}\right.$ for the transition with quantum numbers $7351 \leftarrow 6341$ [15]). There are two close lines near the first frequency $f_{1 \exp }$ in on-line database. These two lines overlap and are detected as one.

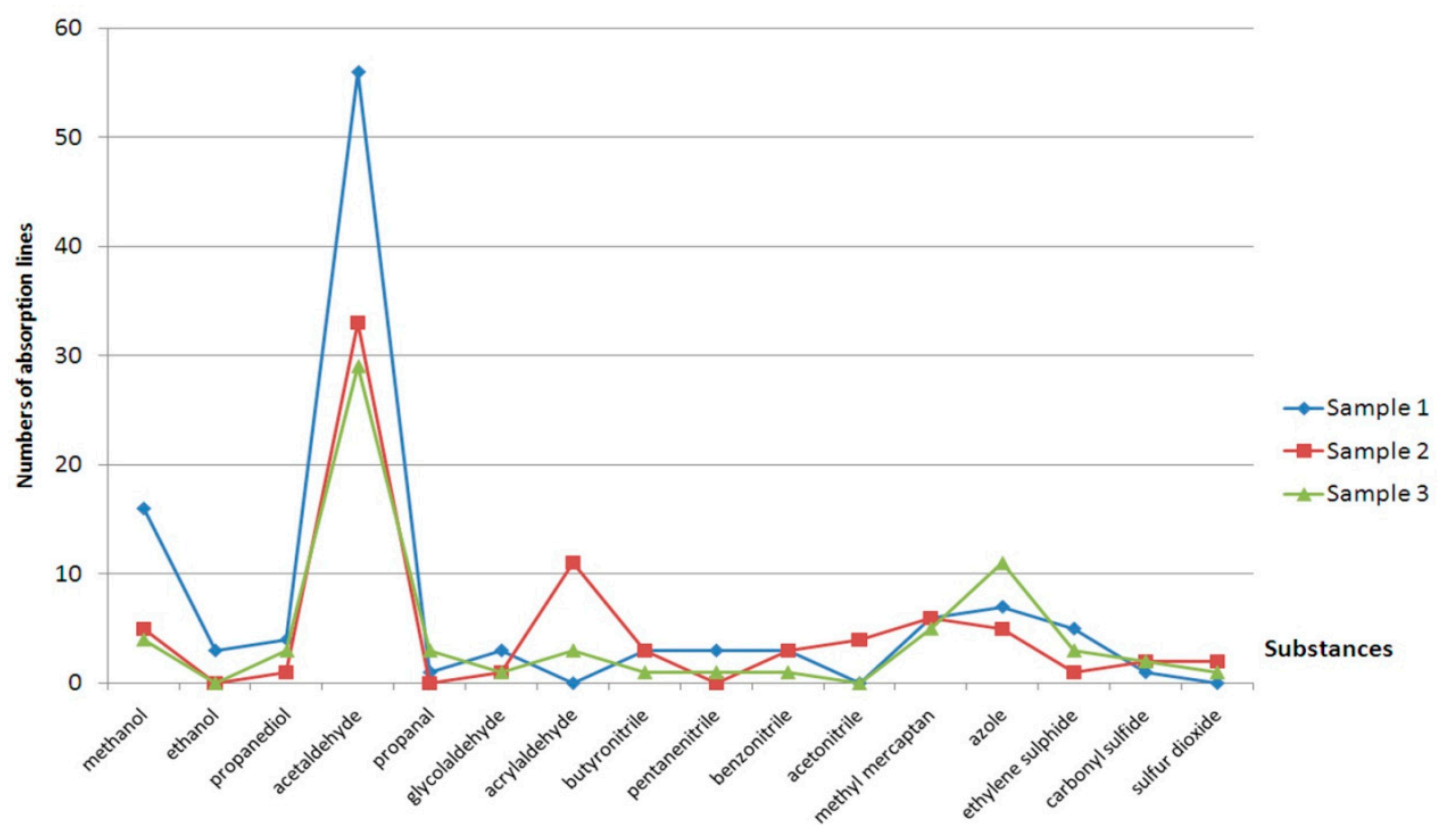

Figure 4. Chemical composition of thermal decomposition products of a relatively healthy nasal mucosa. 


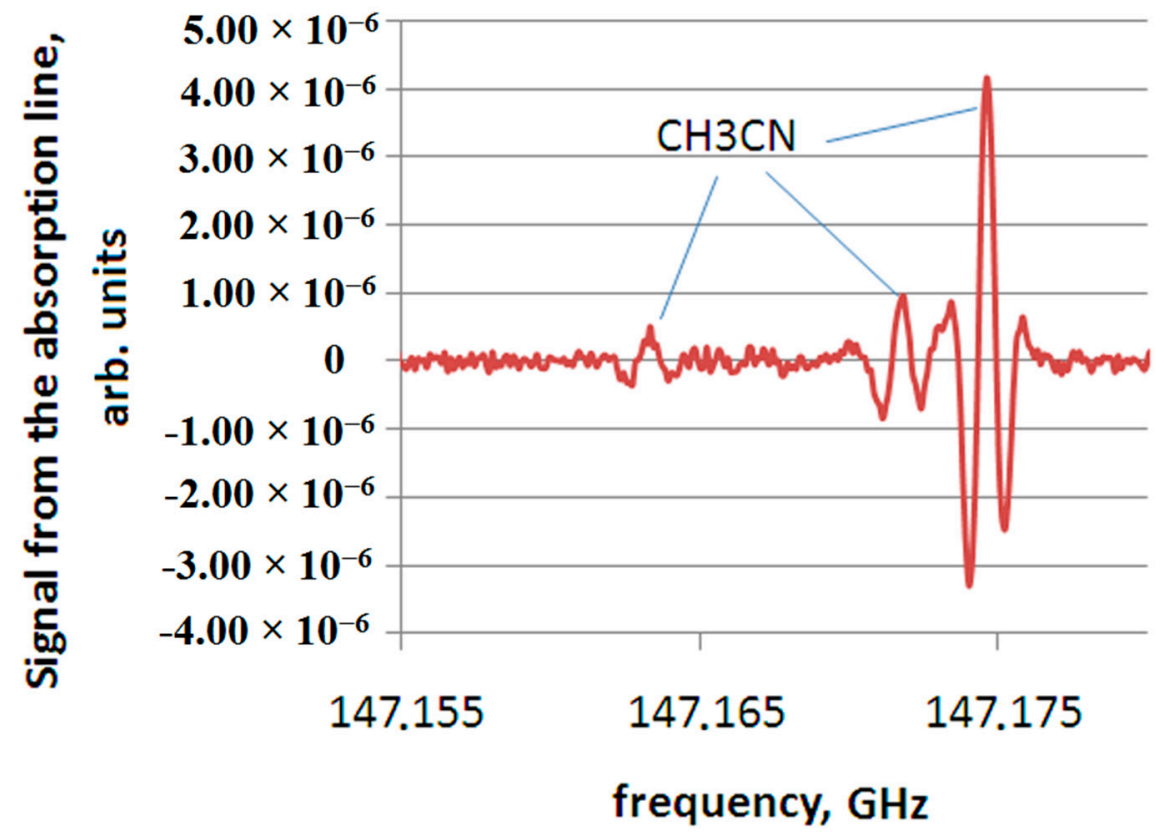

Figure 5. Example of recording a spectrum section containing acetonitrile $\left(\mathrm{CH}_{3} \mathrm{CN}\right)$ lines for a polyp tissue sample (No. 6) with experimentally measured central frequencies $\mathrm{f}_{1 \exp }=147.1633 \mathrm{GHz}\left(\mathrm{f}_{1 \mathrm{cat}}=147.1632441 \mathrm{GHz}\right.$ for a transition with quantum numbers $82 \leftarrow 72$ [15]), $\mathrm{f}_{2 \exp }=147.1718 \mathrm{GHz}\left(\mathrm{f}_{2 \mathrm{cat}}=147.1717519 \mathrm{GHz}\right.$ for a transition with quantum numbers $81 \leftarrow 71$ [15]), and $f_{3 \exp }=147.1746 \mathrm{GHz}\left(\mathrm{f}_{3 \mathrm{cat}}=147.1745883 \mathrm{GHz}\right.$ for a transition with quantum numbers $80 \leftarrow 7$ $0[15])$.

A comparison of the chemical composition of the gas mixture of the thermal decomposition products for polyp and relatively healthy tissue is shown in Figure 6 (polyp tissue Sample No. 6 and healthy tissue Sample No. 3). It can be seen, firstly, that new substances appear in the composition of the gas mixture of the thermal decomposition products for the polyp compared to a relatively healthy mucosa (hydroxyacetone, dihydroxyacetone, propionitrile, including isotopologue with isotope $C$, aminopropionitrile, acetonitrile, monoethanolamine, alanine, pyridine, furan, and phenylacetylene) and the concentration of a number of substances (methyl mercaptan, acetaldehyde, methanol, propanediol, butyronitrile, pentannitrile, azole, diketene, sulfur dioxide, and ethylene sulfide) is increasing.

The composition of the thermal decomposition products of polyp tissue has both a general difference from relatively healthy tissue, and its own characteristics depending on the age of the patient. The etiology of chronic polypous rhinosinusitis with regard to metabolomics is a subject for further study as a part of the research. An example of comparing the chemical composition of thermal decomposition products for two samples (No. 4 for children and No. 5 for adults) is shown in Figure 7.

The figure shows a general coincidence in chemical composition for both samples; however, there are also specific features: the level of acetonitrile and acetaldehyde for a gas mixture of thermal decomposition products of the polyp sample taken from the adult is higher than for the sample taken from the child. 


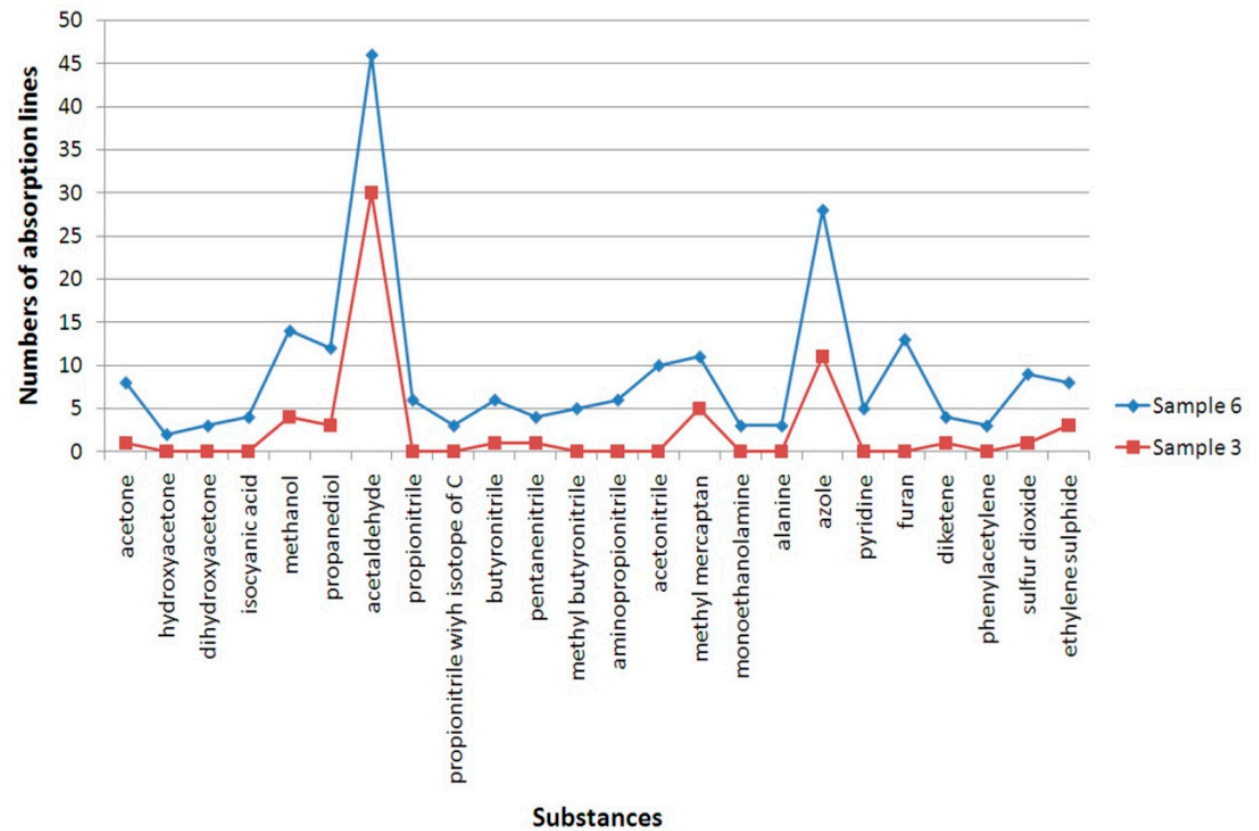

Figure 6. Comparison of the chemical composition of thermal decomposition products of polyp samples (Sample 6) and relatively healthy tissue (Sample 3) taken from a child.

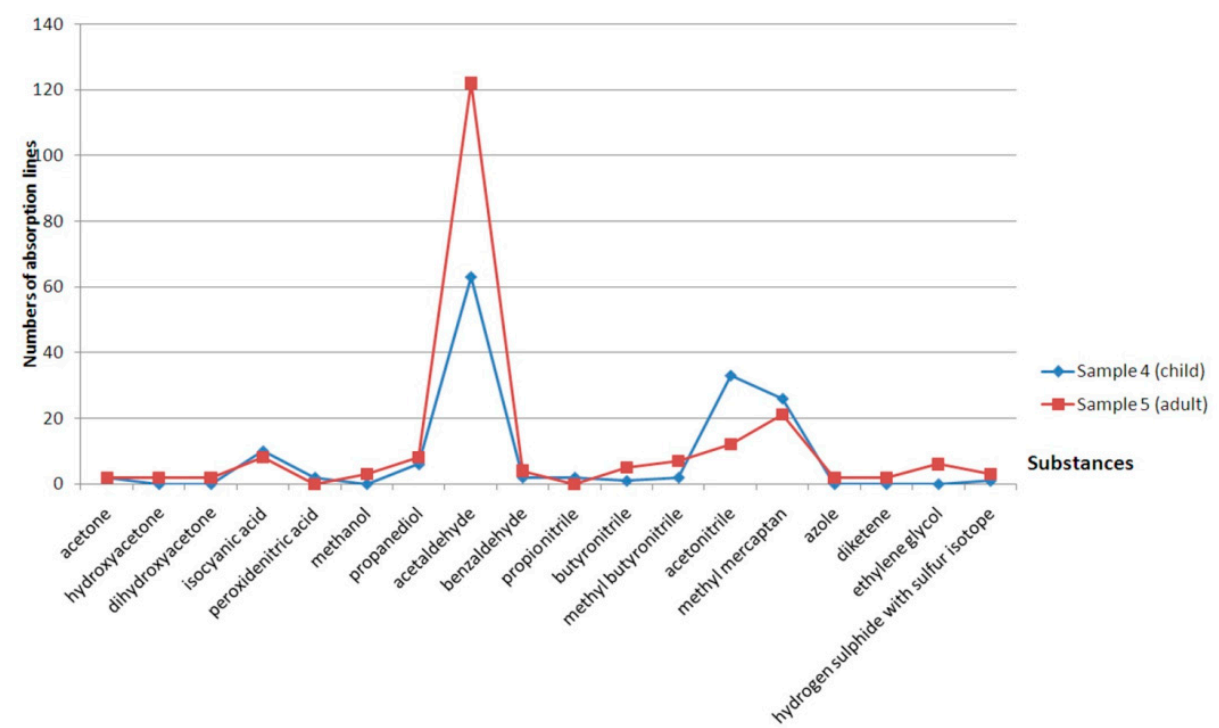

Figure 7. The chemical composition of the products of thermal decomposition of polyp samples taken from a child (Sample 4) and an adult (Sample 5).

\section{Discussion}

The entire metabolites set and the creation of a characteristic metabolic profile with the identification of inflammation markers by the number of absorption lines were analyzed.

Assessment of the precursors of the identified markers allows the biochemical component to be considered as a probable etiological aspect inherent in a specific pathology. For example, acetaldehyde is formed as a result of an oxidative reaction under the action of chemical products of the bacteria vital activity on complex carbohydrates with the formation of pyruvate. The galactose and lactose thermolysis, resulting in the formation of mucic acid and its subsequent reaction with ammonia, can lead to the formation of an azole. Thermal decomposition of cysteine and methionine, as well as of biological tissues as a whole, consisting of complex organic molecules, can lead to the formation of methyl 
mercaptan $[17,18]$. Appearance of other metabolites (e.g., acetonitrile) requires further study and analysis.

Thus, the analysis of tissues metabolites and metabolic processes from the standpoint of biochemistry will reveal possible points of application in the treatment of the disease as a whole at various stages of process development.

\section{Conclusions}

The analysis of the absorption spectra of thermal decomposition products of relatively healthy mucosa and polyps of the nasal cavity and paranasal sinuses has revealed a difference in tissue metabolites. The spectroscopic approach allowed us to detect the most complete set of thermal decomposition products characteristic of this type of tissues, which enables determination of marker substances appearing during the development of pathology.

We have proved the possibility of formation of a metabolic profile as a set of substances detected by high-resolution $\mathrm{THz}$ spectroscopy for a particular pathology confirmed by histomorphological analysis for verification of the considered tissue.

We have identified substances that can be regarded not merely as markers of the corresponding pathology; in addition, their quantitative changes can be used as a factor of the pathogenesis of the disease, with the age of the patient taken into account.

With the accumulation of factual experimental data further study of precursors may become a separate task within the framework of the expanding scientific research.

\section{Patents}

The patent ("A Method of Spectroscopic Investigation of Tissue Metabolites and Device for its Realization"), where the approach to analyzing the products of thermal decomposition of ENT organs tissues reported in this manuscript is presented, is currently at the stage of approval.

Author Contributions: Conceptualization, V.V. and A.A.; methodology, V.V., A.A., S.P. and E.D.; validation, V.V., A.A.; formal analysis, E.D., M.C., A.A., M.S.; investigation, V.A., E.D., M.C.; resources, A.A., K.G., R.L.; data curation, E.D., M.C., A.A.; writing—original draft preparation, M.C., E.D., A.A.; writing-review and editing, M.C., E.D., A.A., V.V.; visualization, E.D., M.C.; supervision, V.V., A.A.; project administration, E.D., A.A.; funding acquisition, V.V. All authors have read and agreed to the published version of the manuscript.

Funding: This research was funded by the Russian Science Foundation, grant No. 21-19-00357.

Institutional Review Board Statement: The study was conducted according to the operational guidelines for ethics committees that review biomedical research (TDR/PRD/ETHICS/2000.1, WHO, Swiss, Geneva, 2000), Consolidated Guideline for Good Clinical Practice (GCP), international ethical guidelines for health-related research involving humans CIOMS (2016) and approved by the Review Board of Otorhinolaringology Department of Privolzhsky Research Medical University (Protocol Code 12 from 10.02.2020).

Informed Consent Statement: Informed consent was obtained from all subjects involved in the study.

Data Availability Statement: Data is contained within the article and is partially available in a publicly accessible repository.

Acknowledgments: The authors acknowledge the contribution of Alexander Nuchev, associate professor of the chemical faculty of the Lobachevsky State University, to the discussion of the experimental results.

Conflicts of Interest: The authors declare no conflict of interest.

\section{References}

1. European Position Paper on Rhinosinusitis and Nasal Polyps. Available online: https://epos2020.com/Documents/supplement_ 29.pdf (accessed on 15 June 2021).

2. Shilenkova, V.V.; Shilenkov, K.A. EPOS-2020. What's new? Russ. Rhinol. 2020, 28, 94-100. (In Russian) [CrossRef] 
3. Kennedy, D.; Sautter, N. Osteitis in chronic rhinosinusitis: A review of the literature. Int. Forum. Allergy Rhinol. $2013,3,355-363$.

4. Fokkens, W.J.; Lund, V.J.; Hopkins, C. European Position Paper on Rhinosinusitis and Nasal Polyps. Rhinology 2020, 58, 1-464. [CrossRef] [PubMed]

5. Wagenmann, M.; Scheckenbach, K.; Chaker, A.M. Endotypes in Chronic Rhinosinusitis: Biomarkers Based on a Mechanistic Insight for Targeted Treatment? ORL J. Otorhinolaryngol. Relat. Spec. 2017, 79, 78-84. [CrossRef] [PubMed]

6. Dobretsov, K.G.; Makarevich, S.V. Morphological assessment of the nasal mucosa in patients with chronic rhinosinusitis with nasal polyps. Russ. Rhinol. 2016, 24, 13-16. (In Russian) [CrossRef]

7. Gowda, G.N.; Zhang, S.; Gu, H.; Asiago, V.; Raftery, N.S.D. Metabolomics-based methods for early disease diagnostics: A Review. Expert Rev. Mol. Diagn. 2008, 8, 617-633. [CrossRef] [PubMed]

8. Amann, A.; de Lacy Costello, B.; Miekisch, W.; Schubert, J.; Buszewski, B.; Pleil, J.; Ratcliffe, N.; Risby, T. The human volatilome: Volatile organic compounds (VOCs) in exhaled breath, skin emanations, urine, feces and saliva. J. Breath Res. $2014,8,034001$. [CrossRef] [PubMed]

9. Wishart, D.S.; Feunang, Y.D.; Marcu, A.; Guo, A.C.; Liang, K.; Vazquez-Fresno, R.V.; Sajed, T.; Johnson, D.; Li, C.; Karu, N.; et al. HMDB 4.0-The Human Metabolome Database for 2018. Nucleic Acids Res. 2018, 46, D608-D617. [CrossRef] [PubMed]

10. Vaks, V.L.; Anfertev, V.A.; Balakirev, V.Y.; Basov, S.A.; Domracheva, E.G.; Illyuk, A.V.; Kupriyanov, P.V.; Pripolzin, S.I.; Chernyaeva, M.B. High resolution terahertz spectroscopy for analytical applications. Physics-Uspekhi 2020, 63, 708-720. [CrossRef]

11. Vaks, V.L.; Domracheva, E.G.; Sobakinskaya, E.A.; Chernyaeva, M.B. Exhaled breath analysis: Physical methods, instruments and medical diagnostics. Physics-Uspekhi 2014, 57, 684-701. [CrossRef]

12. Lykina, A.A.; Anfertev, V.A.; Domracheva, E.G.; Chernyaeva, M.B.; Kononova, Y.A.; Toropova, Y.G.; Korolev, D.V.; Smolyanskaya, O.A.; Vaks, V.L. Terahertz high-resolution spectroscopy of thermal decomposition gas products of diabetic and non-diabetic blood plasma and kidney tissue pellets. J. Biomed. Opt. 2021, 26, 043008.

13. Vaks, V.L.; Brailovsky, A.B.; Khodos, V.V. Millimeter Range Spectrometer with Phase Switching-Novel Method for Reaching of the Top Sensitivity. Infrared Millim. Waves 1999, 20, 883-896.

14. Khodos, V.V.; Ryndyk, D.A.; Vaks, V.L. Fast passage microwave molecular spectroscopy with frequency sweeping. Eur. Phys. J. Appl. Phys. 2004, 25, 203-208. [CrossRef]

15. Pickett, H.M.; Cohen, E.A.; Drouin, B.J.; Pearson, J.C. Submillimeter, Millimeter, and Microwave Spectral Line Catalog. JPL Molecular Spectroscopy. California Institute of Technology. Available online: http://spec.jpl.nasa.gov/ftp/pub/catalog/catform. html (accessed on 10 June 2021).

16. Endres, C.P.; Schlemmer, S.; Schilke, P.; Stutzki, J.; Müller, H.S.P. The Cologne Database for Molecular Spectroscopy, CDMS, in the Virtual Atomic and Molecular Data Centre, VAMDC. Mol. Spectrosc. 2016, 327, 95-104. [CrossRef]

17. "CAS SciFinder" Database. Available online: https:/ / scifinder.cas.org (accessed on 5 July 2021).

18. "Reaxys Chemistry Database". Available online: https://www.reaxys.com (accessed on 5 July 2021). 